\title{
Optical fiber-based liquid level sensor
}

\author{
Sameer M. Chandani \\ Nicolas A. F. Jaeger, MEMBER SPIE \\ University of British Columbia \\ Department of Electrical and Computer \\ Engineering \\ 2332 Main Mall \\ Vancouver, BC, V6T 1Z4, Canada \\ E-mail: sameerc@ece.ubc.ca
}

\begin{abstract}
A liquid level sensor based on etched D-shaped silica optical fibers is presented. The optical transmission of the sensor depends on the liquid level. The sensor can be realized as a continuous level sensor with a high resolution and requires only one fiber and one optical source and detector. We have demonstrated a sensor with a resolution of $\sim 1 \mathrm{~mm}$. The sensor works for liquids with refractive indices greater than $\sim 1.45$. We show that the sensor response is the same for increasing and decreasing liquid levels. ( 2007 Society of Photo-Optical Instrumentation Engineers. [DOI: 10.1117/1.2801506]
\end{abstract}

Subject terms: fiber-optic sensors; D-fibers; liquid level.

Paper 070159RR received Feb. 20, 2007; revised manuscript received Jun. 6, 2007; accepted for publication Jun. 8, 2007; published online Nov. 13, 2007.

\section{Introduction}

Optical fiber-based sensors have been the subject of intense research activity for more than three decades. ${ }^{\text {. Such sensors }}$ offer two main advantages; they are made from nonconducting (dielectric) materials and they are corrosion resistant. The former advantage is particularly attractive for applications in explosive environments and flammable atmospheres while the latter is attractive for chemical processing applications. An application that is well served by these two advantages is in liquid level sensing of flammable hydrocarbons or fuels and of chemicals in industrial and processing plants. For these reasons, there has been a significant amount of research into optical liquid level sensors. $^{2-17}$

Liquid level sensors can be classified into two main categories: continuous level sensors and discrete level sensors. Several continuous level sensors have been demonstrated. The coupling between optical waveguides at the surface of a liquid using six fibers and a reflecting array comprised of steel wires was demonstrated in Ref. 2. A sensor using two specially treated plastic optical fibers immersed in a quartz tube filled with an intermediate liquid was developed in Ref. 3. Some systems measure the reflected light off of the surface of the liquid using multiple fibers with collimating optics $^{4,5}$ or ladar techniques, ${ }^{6-8}$ to measure the liquid surface proximity. Such systems require the placement of transmitting and receiving fibers and/or collimating optics at a fixed point above the liquid surface. The light can undergo undesired reflections or refractions due to ripples on the liquid's surface leading to errors. The refractive index sensitivity of long-period fiber-optic gratings has been exploited in Ref. 9, but suffered limitations such as the need to use a specific wavelength, a linear response over only about $60 \%$ of the grating's length and a limiting restriction on the liquid's refractive index (restricted to the range 1.400-1.456). Most recently, a sensor based on the optical attenuation in a plastic optical fiber where the liquid acted as a second cladding was demonstrated in Ref. 10 with a resolution of $2 \mathrm{~mm}$.

0091-3286/2007/\$25.00 @ 2007 SPIE
Discrete level sensors are typically based on the change in optical reflection from or transmission through the sensor head, due to the change in refractive index surrounding the sensor head, when immersed in a liquid. Examples of such sensors are based on the coupling of cladding modes from a lead-in fiber to a lead-out fiber, ${ }^{1 \uparrow}$ on frustrated total internal reflection experienced in fibers with angled or retroreflecting tips ${ }^{12}$ or conical/tapered tips, ${ }^{13}$ on total internal reflection experienced in fibers with reflecting elements such as prisms ${ }^{14}$ or specially designed tips ${ }^{15}$ cemented to their ends, and most recently on transmission through two fibers integrated using a hemispherical transparent dielectric element. ${ }^{16}$ In each of the above cases, the sensor has been implemented as an on-off switch, or as an array of sensors placed along the wall of the liquid vessel, or on a mechanical assembly that translated the sensor up and down the liquid vessel. Thus, to achieve high resolutions, an array of many sensors (e.g., 140 in Ref. 16) or some additional moving mechanism is needed, which adds to the cost and complexity of the level measurement.

Betta et al. have demonstrated a quasi-continuous sensor based on "unclad zones" on a plastic clad silica fiber where the cladding has been reduced in these zones to cause optical attenuation when surrounded by a liquid. ${ }^{17}$ Using six fibers, each with 63 unclad zones, a $1 \mathrm{~m}$ full-scale, $4 \mathrm{~mm}$ resolution prototype was realized and tested. The sensor length is limited due to the fact that the power attenuation of each unclad zone is less than that of the previous zone so that, as the liquid level rises, the change in optical power becomes comparable to the noise amplitude.

In this paper we describe and demonstrate a liquid level sensor that is based on power attenuation experienced in etched D-shaped optical fibers. The sensor can be realized as a continuous level sensor or as a discrete level sensor. In either case the use of only one fiber, one optical source and one detector is needed.

\section{Operating Principle}

In a typical step-index single-mode optical fiber the refractive index of the core, $n_{\mathrm{co}}$, is greater than that of the cladding, $n_{\mathrm{cl}}$, and total internal reflection at the core-cladding boundary keeps the light guided. When the cladding thick- 


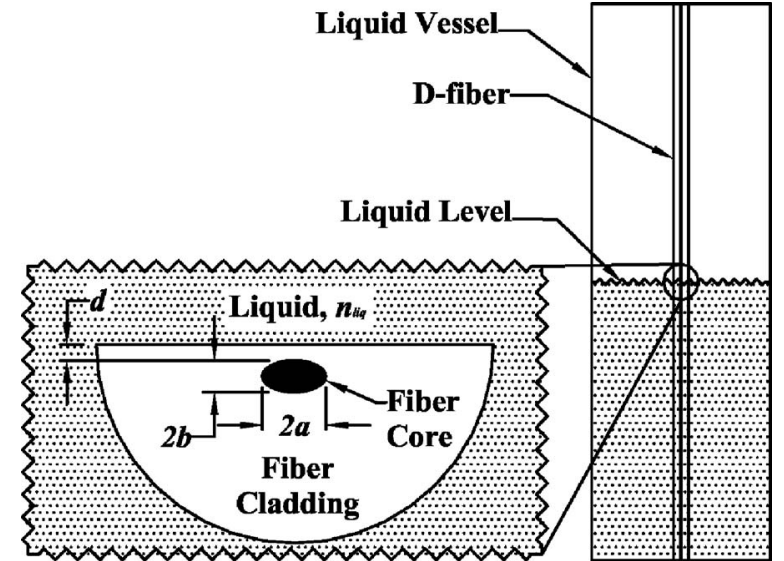

Fig. 1 Conceptual diagram of liquid level sensor with cross section of sensor head.

ness is reduced (typically to less than $10 \mu \mathrm{m}$ ) and a second cladding comes into contact with the first cladding, the fiber's fundamental mode over the region that is in contact is affected. In particular, if the refractive index of the second cladding is less than the mode's effective index, $n_{\text {eff }}$ $\left(=\beta / k_{o}\right)$, the propagation constant, $\beta$, is changed and the fiber continues to guide light. On the other hand, if the refractive index of the second cladding is greater than $n_{\mathrm{eff}}$, the mode becomes leaky and the propagation constant of the mode becomes complex, i.e., $\beta=\beta_{r}+j \beta_{j}$, leading to an attenuation of the power launched into the fiber. ${ }^{1822} \mathrm{Re}$ ducing the cladding thickness over a long length can easily be achieved with D-shaped optical fibers, while still retain- ing the mechanical strength of the fiber. The cladding is typically reduced by etching the fiber in hydrofluoric acid.

A liquid level sensor can be realized by using the liquid as the second cladding of such a "reduced-cladding" D fiber. The power attenuation coefficient, $\gamma=2 \beta_{j}$, of the etched section of the $\mathrm{D}$ fiber (which constitutes the sensor head) that is in contact with the liquid, depends on the fiber core's dimensions, on its mode effective index, on its cladding thickness, $d$ (defined as the minimum distance between the core edge and the fiber flat), on the operating wavelength, $\lambda_{o}$, and on the refractive index of the liquid, $n_{\text {liq. }}$. The D fibers used here (obtained from KVH Industries) were single-mode polarization maintaining and had elliptical cores with $n_{\mathrm{co}}=1.4756$ and $n_{\mathrm{cl}}=1.4410$ at $\lambda_{o}$ $=1550 \mathrm{~nm}$. Figure 1 shows a conceptual diagram of the liquid level sensor and the cross section of the D fiber used.

The sensor head, using a single-mode step-index elliptical core fiber, surrounded by the liquid can be theoretically modeled as described in Ref. 23 and its power attenuation coefficient can be determined analytically for $n_{\text {liq }}>n_{\text {eff }}$. Figure 2 shows the calculated power attenuation coefficient for the elliptical D-fiber's fundamental mode (the ${ }_{0} \mathrm{HE}_{11}$ mode as defined in Refs. 24 and 25) as a function of the liquid refractive index for four values of the cladding thickness at $\lambda_{o}=1550 \mathrm{~nm}$. The power attenuation coefficient peaks sharply close to the fiber mode's effective index and then monotonically decreases for larger refractive indices. For $n_{\mathrm{liq}}<n_{\mathrm{eff}}$, the power attenuation coefficient, $\gamma$, is zero and there is no power lost from the sensor head due to contact with the liquid (as is typical with optical fibers, there are intrinsic losses due to other mechanisms such as scattering and absorption; however, these are negligible over short lengths). The power attenuation coefficient
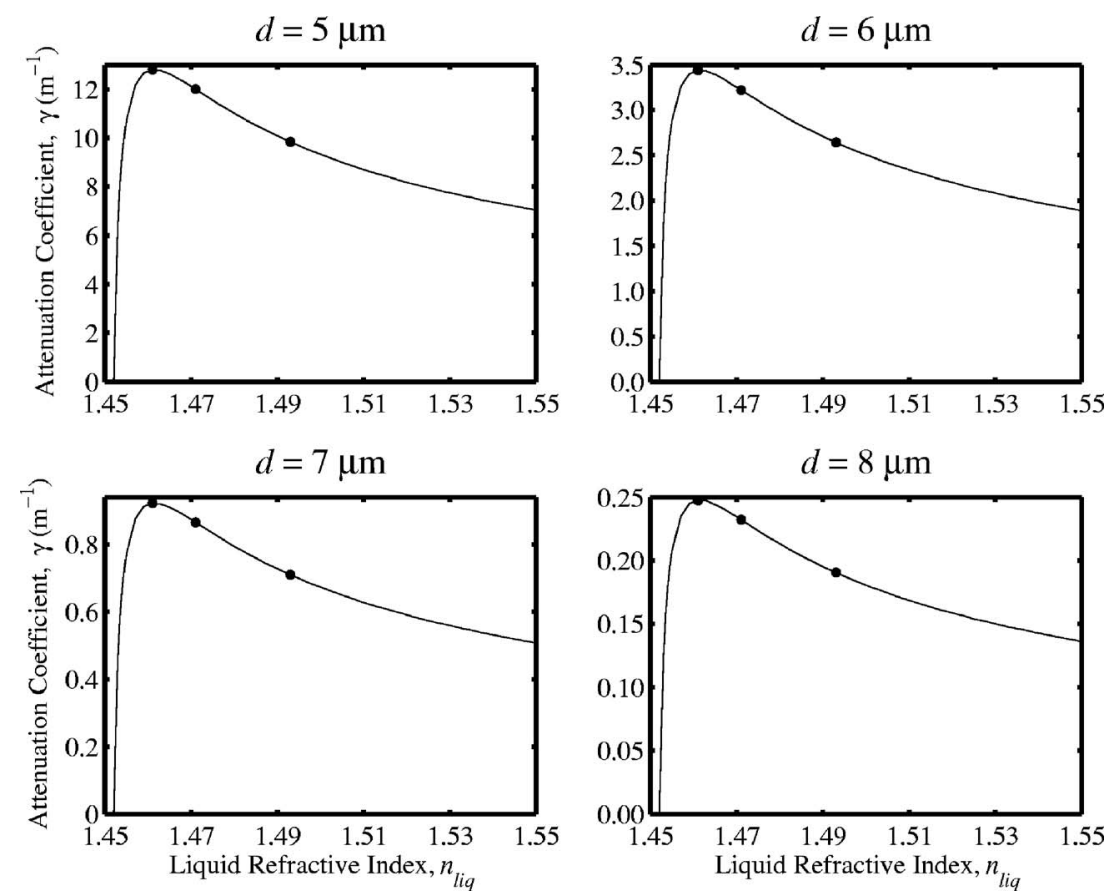

Fig. 2 Calculated power attenuation coefficient for the ${ }_{0} \mathrm{HE}_{11}$ mode as a function of liquid refractive index for four values of $d$ using $a=2 \mu \mathrm{m}, b=1 \mu \mathrm{m}$ and $\lambda_{o}=1550 \mathrm{~nm}$. Solid circles indicate points of interest. 

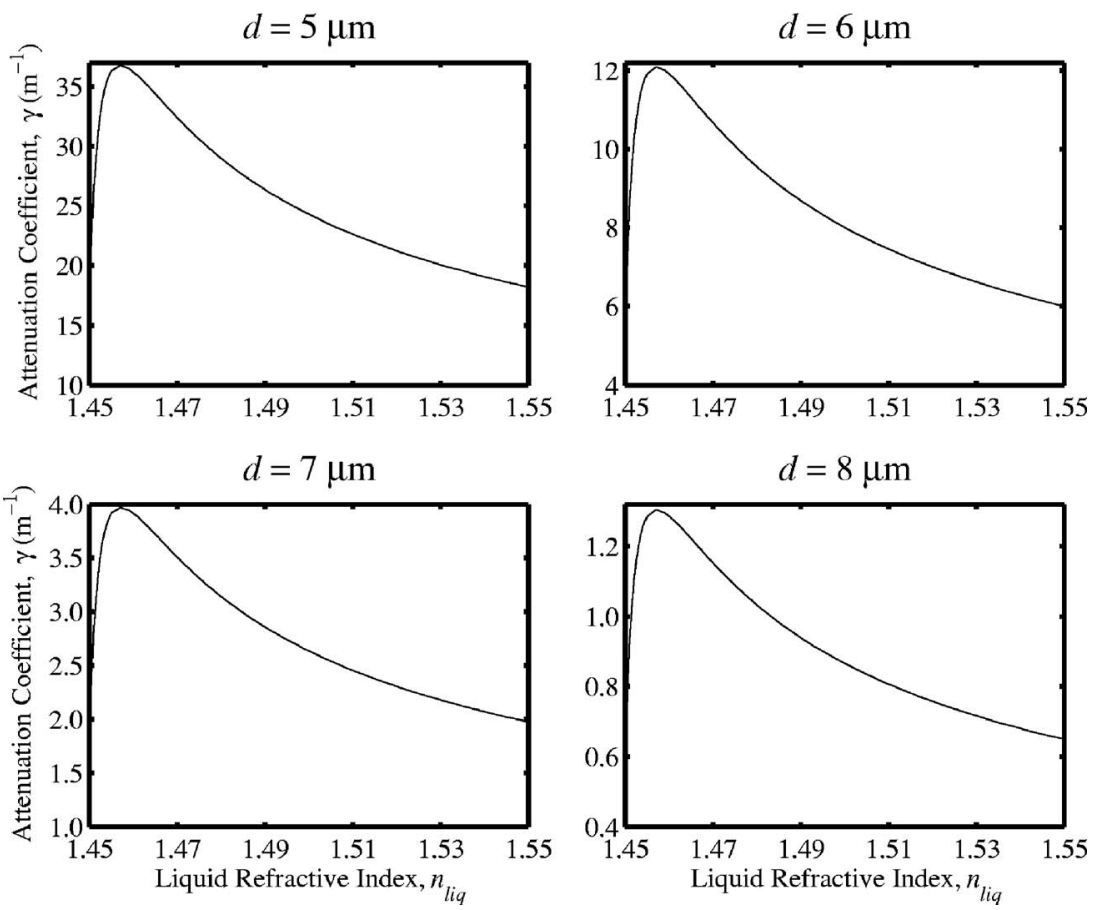

Fig. 3 Calculated power attenuation coefficient for the ${ }_{0} \mathrm{HE}_{11}$ mode as a function of liquid refractive index for four values of $d$ using $a=2 \mu \mathrm{m}, b=1 \mu \mathrm{m}$ and $\lambda_{o}=1650 \mathrm{~nm}$.

curves can be shifted to the left by increasing $\lambda_{o}$. For example, Fig. 3 shows the calculated attenuation using $\lambda_{o}=1650 \mathrm{~nm}$. It is apparent from Fig. 2 and Fig. 3 that the proposed sensor can be designed and operated such that it can be used with any liquid for which $n_{\text {liq }}>1.45$. This is a favorable result considering that the refractive indices of many liquid hydrocarbons and other industrial liquids are in the range from 1.45 to $1.55 .^{3,26}$ Changes in the liquid refractive index (for example, due to temperature fluctuations) could lead to errors since the attenuation coefficient is sensitive to the liquid refractive index. Nevertheless, in an industrial application, compensation techniques commonly used with intensity-based optical fiber sensors can be applied. ${ }^{27}$

The power transmission ratio, $T_{P}$, of the sensor head can be written as

$T_{P}=\frac{P_{\text {out }}}{P_{\text {in }}}=e^{-2 \beta_{j} L}=e^{-\gamma L}$,

where $P_{\text {in }}$ and $P_{\text {out }}$ are the optical powers into and out of the sensor head, respectively, and $L$ is the length of the sensor head that is surrounded by the liquid. For example, Eq. (1) has been used to calculate the sensor's power transmission ratio at $\lambda_{o}=1550 \mathrm{~nm}$ and is shown in Fig. 4 as a function of the liquid level for several values of $n_{\text {liq }}$ using $d=8 \mu \mathrm{m}$. Here, a cladding thickness of $8 \mu \mathrm{m}$ was chosen to give a nearly linear response over the length of the sensor. The sensitivity of the sensor, i.e., the slope of the response $\left(\partial T_{p} / \partial L\right)$, depends on $\gamma$ and thus, depending on the application, the sensor response and its sensitivity can be tailored by carefully selecting the cladding thickness to yield a desired value for $\gamma$ for a particular $n_{\text {liq. }}$. It should be noted that the average slope of the response for a particular $d$ decreases as $n_{\text {liq }}$ increases. In general, it is not necessary to have a linear sensor. For a given length of sensor, if the power attenuation coefficient is high (due to a smaller cladding thickness), the sensor response becomes exponential and remains relatively linear for low liquid levels. In addition, the sensitivity decreases with increasing liquid levels for such an exponential sensor. Nevertheless, such a sensor may be advantageous for applications requiring a high sensitivity for low liquid levels and a lower sensitivity for high liquid levels.

The sensor can also be realized as a discrete level sensor for applications that have high liquid levels and require low resolutions. Short sections of the fiber (e.g., $1 \mathrm{~mm}$ ) could

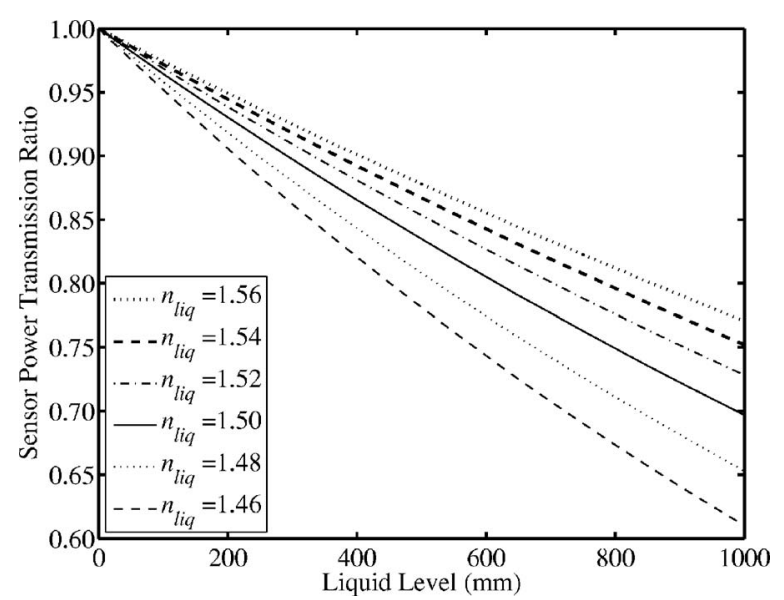

Fig. 4 Calculated liquid level sensor power transmission ratio as a function of liquid level for several values of $n_{\text {liq }}$ using $d=8 \mu \mathrm{m}$, $a=2 \mu \mathrm{m}, b=1 \mu \mathrm{m}$ and $\lambda_{o}=1550 \mathrm{~nm}$. 


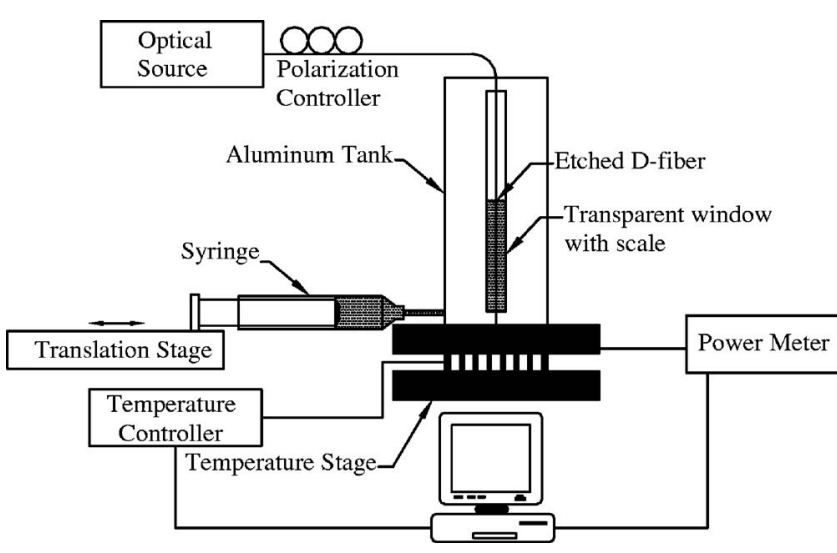

Fig. 5 Experimental setup showing $D$ fiber running down center of aluminum tank with sensor head in the middle of tank. The $1550 \mathrm{~nm}$ optical source is used to couple light via a polarization controller into the sensor. The syringe on the translation stage is used to change the liquid level. The computer remotely controls all the instruments and acquires data from the power meter.

be etched at regular intervals (determined by the required resolution) so that they all yield the same attenuation and the sensor response would then be a stair-like function, each step corresponding to a discrete liquid level. Such a discrete sensor requiring only one fiber, one source and one detector results in a significant decrease in the complexity and cost as compared to existing discrete level sensors that require multiple sensors, lead-in and lead-out fibers, sources and detectors $^{11,14,16}$ or moving mechanisms. ${ }^{12,13,15}$

\section{Sensor Fabrication and Characterization}

To fabricate the proposed sensor, a $19-20 \mathrm{~mm}$ unjacketed section of our D fiber was etched in a $10 \%$ hydrofluoric acid solution for $\sim 170 \mathrm{~min}$ to decrease the cladding thickness to between 5 and $6 \mu \mathrm{m}$. As will be seen later, this was done to achieve a power attenuation coefficient that would result in a linear sensor response over its length. Standard thermo-optic oils (obtained from Cargille Labs) were used as the liquids so that their temperatures could be used to accurately control their refractive indices.

The experimental setup in Fig. 5 shows the D fiber running down the center of a specially built square aluminum tank, which had a transparent window along one of its walls with a scale, in $\mathrm{mm}$, to read the liquid level. A plugged hole at the bottom of the tank allowed the routing of the fiber to the power meter. Alternatively, as in Ref. 10, the fiber can be looped back to the top of the tank so as to make a "U" shape. In this case, however, only one side of the U shape needs to be etched to create the sensor head. The other side of the $U$ shape serves to direct the modulated light back to the power meter with negligible power loss. The liquid was inserted into the tank using a syringe. To accurately control the liquid level (by either introducing or extracting a small amount of liquid) the end of the syringe's plunger was attached to an automated translation stage. The aluminum tank was placed on a temperature stage so that the liquid's temperature, and hence its refractive index, could be controlled throughout the experiment. A $1550 \mathrm{~nm}$ monochromatic optical source was used and the light was coupled into the sensor from a standard SMF-28 fiber using a mechanical splice. A polarization controller was inserted between the optical source and the sensor so that the fundamental ${ }_{0} \mathrm{HE}_{11}$ mode of the $\mathrm{D}$ fiber could be launched. This mode has its electric field polarized parallel to the major axis of the elliptical core. ${ }^{24,25}$ The reason for launching this mode is that the theoretical model used was developed for this mode. To confirm that the ${ }_{0} \mathrm{HE}_{11}$ mode was launched, a polarizer placed at the output was used to measure the extinction ratio of the light, which was always in excess of $27 \mathrm{~dB}$. The output of the fiber was directed onto a power meter. The computer shown in Fig. 5 was used to remotely control the temperature controller, polarization controller, and translation stage and to acquire data from the power meter. To measure the power transmission ratio, the output power of the sensor was recorded with a liquid level of zero, i.e., air surrounding the sensor head. This power level was used as the reference to calculate the power transmission ratio so that for a liquid level of zero, $T_{P}=1$. Thus, when the liquid level increases, the power transmission ratio decreases and thus becomes less than one. In this way, we normalize out the power losses due to other mechanisms such as intrinsic attenuation and absorption in the D fiber.

The characterization of the sensor was split into (1) static characterization and (2) dynamic characterization.

\subsection{Static Characterization of the Sensor}

The static characterization was carried out by measuring the sensor's response to increasing and decreasing liquid levels, which were changed in $2 \mathrm{~mm}$ steps. At each liquid level, the power level was measured and the power transmission ratio was calculated as described earlier in this section. The response was measured at three values of refractive index achieved using two oils. OIL-A had an index of refraction at $25^{\circ} \mathrm{C}, n_{D}$ and thermo-optic coefficient, $d n_{D} / d T$, of 1.471 and $-3.86 \times 10^{-4} /{ }^{\circ} \mathrm{C}$, respectively, and OIL-B had $n_{D}=1.493$ and $d n_{D} / d T=-3.91 \times 10^{-4} /{ }^{\circ} \mathrm{C}$, all at $\lambda_{o}=1550 \mathrm{~nm}$. The third refractive index was achieved by using OIL-A at $50{ }^{\circ} \mathrm{C}$ which resulted in an index of refraction of 1.461 . These three values were chosen because they approximately occur at points of interest on the power attenuation coefficient curve of our D fiber (indicated by solid circles in Fig. 2), i.e., $n_{\text {liq }}=1.461$ occurs near the peak of the curve, $n_{\text {liq }}=1.471$ occurs near where the slope of the curve to the right of its peak is steepest and $n_{\text {liq }}=1.493$ occurs near the middle of the range $1.45-1.55$ for typical hydrocarbons and other industrial liquids. Figures 6-8 show the sensor's measured power transmission ratio using liquids with the three refractive index values above. Shown in each figure is the response to an increasing and a decreasing liquid level. Each response has been fit to a line to illustrate the sensor's linearity with the fitted line's slope, $m$, and regression coefficient, $R$, given in the legend. The calculated slopes for the increasing and decreasing liquid levels differ by $\sim 1 \%$ in each case, demonstrating that the sensor has virtually identical responses for increasing and decreasing liquid levels. The regression coefficients for the linear fits are all greater than 0.99 signifying the sensor's linearity. As predicted, the value of the slope decreases with increasing liquid refractive index.

The resolution of the sensor depends on the measurement of the optical transmission, which can fluctuate due to 


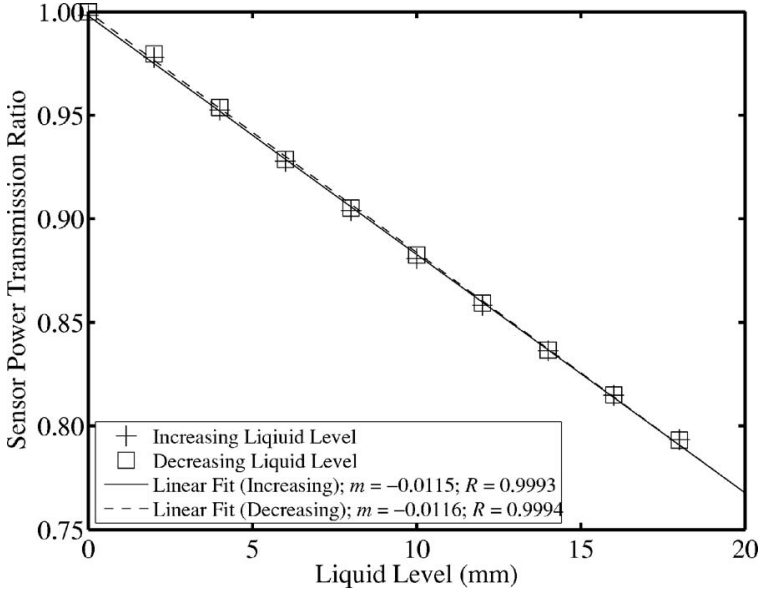

Fig. 6 Sensor's measured power transmission ratio vs liquid level for $n_{\text {liq }}=1.461$ for increasing and decreasing liquid levels along with linear fits.

factors such as source temperature changes, detector responsivity changes, link attenuation changes, and those caused by changes in mechanical components such as connectors, mechanical support mounts, and optics. ${ }^{28}$ A conservative optical transmission measurement resolution of $1 \%$ yields a liquid level measurement resolution of $\sim 1 \mathrm{~mm}$.

\subsection{Dynamic Characterization of the Sensor}

The dynamic characterization of the sensor was carried out by continuously varying the liquid level at a constant rate. The liquid level was first increased at a particular rate to fill up the tank, thus immersing the entire sensor head. After some time, the level was then decreased at the same rate. This time interval was introduced to demonstrate the power transmission ratio's stability while the liquid level remained stationary. As the liquid level increased or decreased with time, the sensor's power transmission was sampled every second to calculate the corresponding power transmission ratio. The sensor's response to a dynamically changing liquid level is shown in Fig. 9 for $n_{\text {liq }}=1.461$ and in Fig. 10

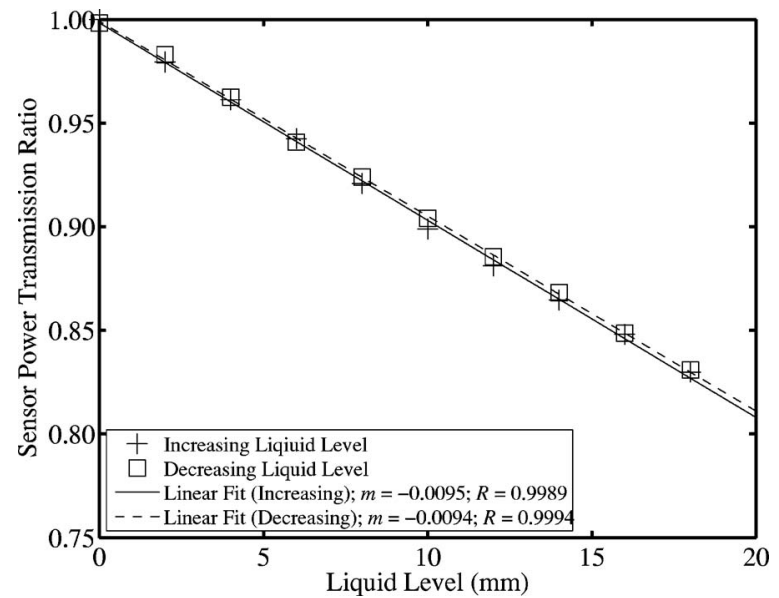

Fig. 7 Sensor's measured power transmission ratio vs liquid leve for $n_{\text {liq }}=1.471$ for increasing and decreasing liquid levels along with linear fits.

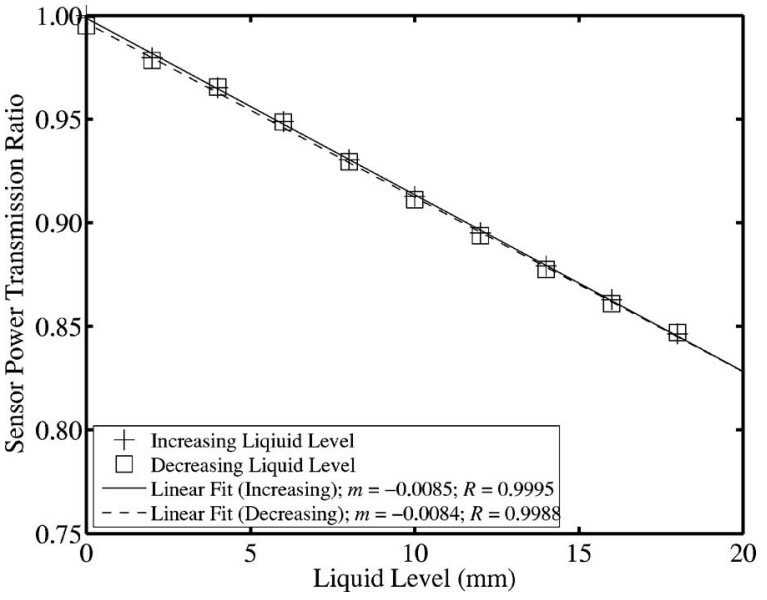

Fig. 8 Sensor's measured power transmission ratio vs liquid level for $n_{\text {liq }}=1.493$ for increasing and decreasing liquid levels along with linear fits.

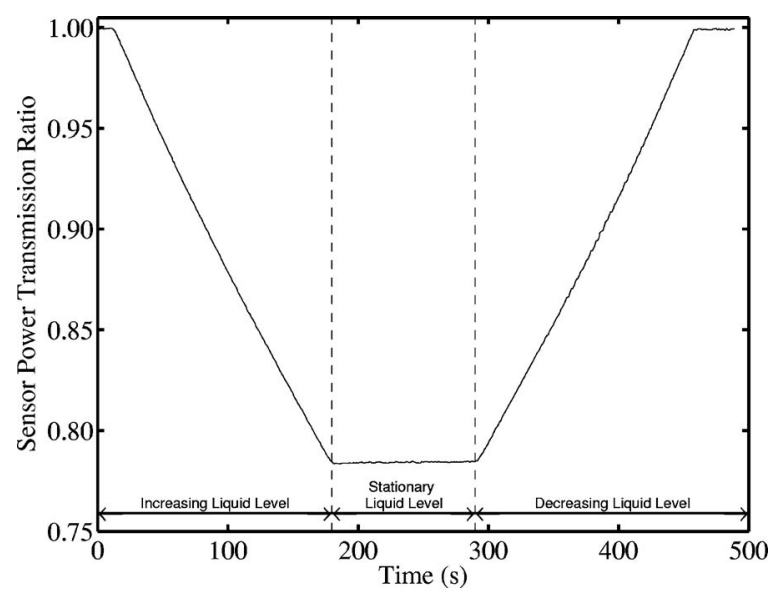

Fig. 9 Sensor's measured power transmission ratio as a function of time for increasing and decreasing liquid levels for $n_{\text {liq }}=1.461$.

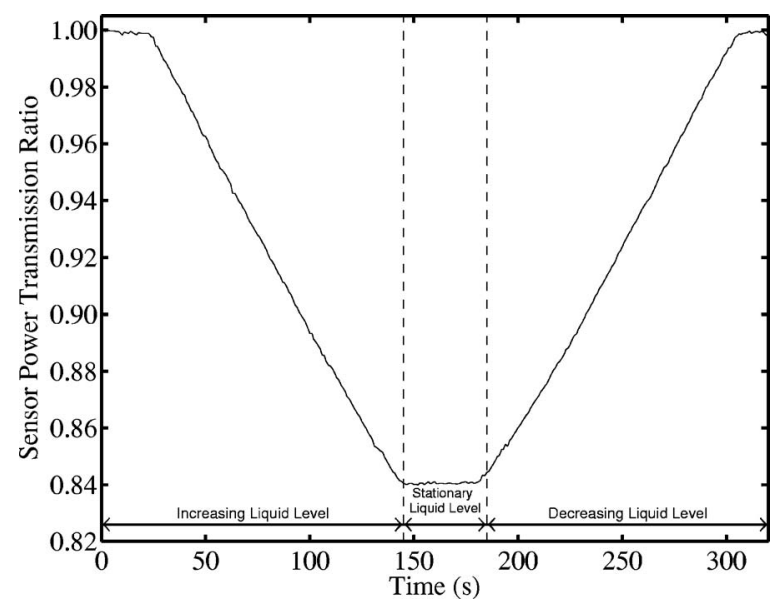

Fig. 10 Sensor's measured power transmission ratio as a function of time for increasing and decreasing liquid levels for $n_{\text {liq }}=1.493$. 


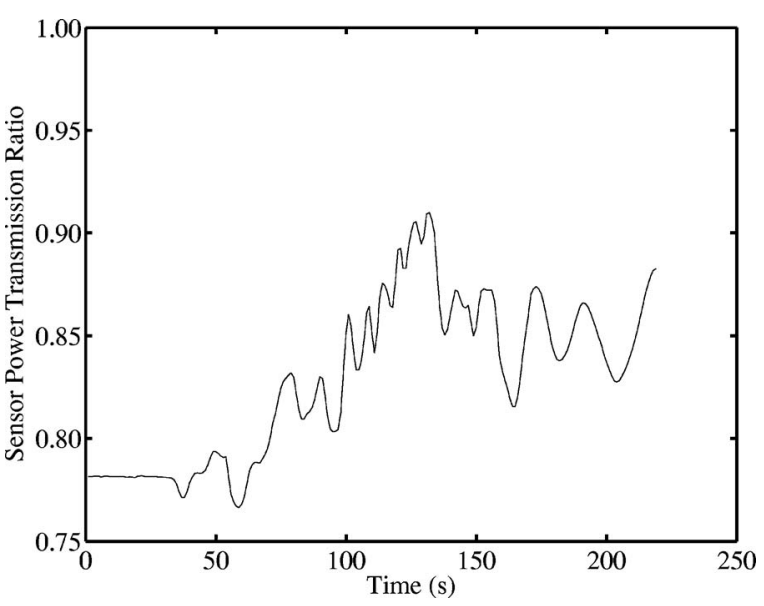

Fig. 11 Sensor's measured power transmission ratio as a function of time with liquid level decreasing at a rapid rate for $n_{\text {liq }}=1.461$.

for $n_{\mathrm{liq}}=1.493$. As seen, the power transmission ratio decreases linearly with increasing liquid level and increases linearly back to one with decreasing liquid level. The dashed vertical lines in the figures separate the three different regions described above.

The rate of change of the liquid level only affects decreasing liquid levels. With increasing liquid levels, the rate is only limited by the optical detection time. However, if the level is decreased rapidly, the sensor's power transmission ratio does not increase linearly, as expected, but fluctuates with time. In fact, in some cases the power transmission ratio decreases to values below the minimum power transmission ratio value (i.e., the power transmission ratio value when the entire sensor head is immersed in the liquid). Figure 11 shows an example of the sensor's measured power transmission ratio with a rapidly decreasing liquid level for $n_{\text {liq }}=1.461$. These fluctuations in the sensor transmission ratio are likely due to a thin film of liquid being left behind on the sensor head. It is possible that such a film would likely form a planar waveguide which would interact with, i.e., couple with, the core of the D fiber. ${ }^{20,29,30}$ Such coupling would likely be strongly dependent on the thickness and length of the liquid film, both of which would likely change over time, leading to oscillations in the sensor transmission ratio.

\section{Conclusions}

We have demonstrated a liquid level sensor using etched D-shaped silica optical fibers. Once calibrated, our sensor has a resolution of $1 \mathrm{~mm}$ when used as a continuous level sensor and had virtually the same response for increasing and decreasing levels.

Advantages of the reported sensor over other continuous level sensors that use an optical fiber immersed in the liquid $^{2,3,17}$ is that it only requires one piece of fiber to achieve a high resolution. In Ref. 10 where one plastic optical fiber has been used with the liquid acting as a second cladding, the refractive index of the liquid has to be less than the refractive index of the fiber (typically $\sim 1.49$ for plastic fibers) to cause total internal reflection, thus placing an upper limit on the liquid's refractive index. The sensor based on the refractive index sensitivity of long- period fiber gratings ${ }^{9}$ has a higher resolution; however, its operating principle is far more complicated, its refractive index range is limited to $1.400-1.456$, it requires the use of a specific wavelength and it has a linear response over only about $60 \%$ of the grating's length.

The sensor reported here does not require the placement of any collimating optics, or receiving and transmitting fibers above the liquid surface as is required in Refs. 4-8. A simple mechanical splice between a standard single-mode polarization maintaining fiber and the D fiber is all that would be required. The sensor can also be implemented as a discrete level sensor requiring only one fiber, one source and one detector, unlike other discrete level sensors that require multiple sensors. ${ }^{11-16}$

The range of operation of the sensor is only limited by the length of the sensor, where a longer sensor would lead to an exponential response. This is not a serious limitation as a linear response is not necessary if appropriately calibrated. However, the sensitivity of the sensor would be less. Careful selection of the power attenuation coefficient can lead to a nearly linear response over the required length of the sensor. The refractive index of the liquid must be greater than the mode effective index of the fiber, which in our case was $\sim 1.45$. Since the liquid is in contact with the fiber, only liquids that do not modify the fiber should be used. Rapidly decreasing liquid levels can lead to errors in the sensor's response, which is likely due to residual liquid being left on the fiber surface.

\section{Acknowledgment}

This work was supported in part by the National Science and Engineering Council of Canada.

\section{References}

1. S. D. Crossley, "The Commercialization of fiber optic sensors," Chapter 2 in Handbook of Optical Fiber Sensing Technology, J. M. López-Higuera, Ed., p. 23, Wiley, West Sussex, United Kingdom (2002).

2. J. A. Morris and C. R. Pollock, "A digital fiber-optic liquid level sensor," J. Lightwave Technol. 5(7), 920-925 (1987).

3. L. A. Danisch, "Removing index of refraction constraints in the optical measurement of liquid level," Proc. SPIE 1795, 268-279 (1993).

4. K. Iwamoto and I. Kamata, "Liquid-level sensor with optical fibers," Appl. Opt. 31(1), 51-54 (1992).

5. C. Vázquez, A. B. Gonzalo, S. Vargas, and J. Montalvo, "Multi-sensor system using plastic optical fibers for intrinsically safe level measurements," Sens. Actuators, A A116(1), 22-32 (2004).

6. D. A. Jackson, "High precision remote liquid level measurement using a combination of optical radar and optical fibers," in Proc. First Int. IEE Conf. Optical Fiber Sensors, pp. 100-103, London, England (1983).

7. W. Peng and J. Lin, "The study on Ladar fiber optical liquid level sensor system," Proc. SPIE 3740, 488-491 (1999).

8. L. Yan-Bing and Y. Yong-Hua, "Optical remote measurement of liquid level," Proc. SPIE 2101, 1004-1007 (1993).

9. S. Khaliq, S. W. James, and R. P. Tatam, "Fiber-optic liquid-level sensor using a long-period grating," Opt. Lett. 26(16), 1224-1226 (2001).

10. F. Pérez-Ocón, M. Rubiño, J. M. Abril, P. Casanova, and J. A. Martínez, "Fiber-optic liquid-level continuous gauge," Sens. Actuators, A A125(2), 124-132 (2006).

11. A. Wang, M. F. Gunther, K. A. Murphy, and R. O. Claus, "Fiber-optic liquid-level sensor," Sens. Actuators, A A35(2), 161-164 (1992).

12. I. K. Ilev and R. W. Waynant, "All-fiber-optic sensor for liquid level measurement," Rev. Sci. Instrum. 70(5), 2551-2554 (1999).

13. L. Ren and Q. Yu, "High-accuracy fiber optic level sensor," Proc. SPIE 4920, 362-366 (2002).

14. C. Yang, S. Chen, and G. Yang, "Fiber optical liquid level sensor under cryogenic environment," Sens. Actuators, A A94(1), 69-75 (2001). 
15. P. Raatikainen, I. Kassamakov, R. Kakanakov, and M. Luukkala, "Fiber-optic liquid-level sensor," Sens. Actuators, A A58(2), 93-97 (1997).

16. K. E. Romo-Medrano and S. N. Khotiaintsev, "An optical-fiber refractometric liquid-level sensor for liquid nitrogen," Meas. Sci. Technol. 17(5), 998-1004 (2006).

17. G. Betta, A. Pietrosanto, and A. Scaglione, "A Gray-code-based fiber optic liquid level transducer," IEEE Trans. Instrum. Meas. 47(1) 174-178 (1998)

18. J. A. Arnaud, "Transverse coupling in fiber optics, Part II: Coupling to mode sinks," Bell Syst. Tech. J. 53(4), 675-696 (1974)

19. J. A. Arnaud, "Transverse coupling in fiber optics, Part I: Coupling between trapped modes," Bell Syst. Tech. J. 53(2), 217-224 (1974).

20. D. Marcuse, "Investigation of coupling between a fiber and an infinite slab," J. Lightwave Technol. 7(1), 122-130 (1989).

21. M. J. F. Digonnet, J. R. Feth, L. F. Stokes, and H. J. Shaw, "Measurement of the core proximity in polished fiber substrates and couplers," Opt. Lett. 10(9), 463-465 (1985).

22. S. M. Chandani and N. A. F. Jaeger, "Fiber-optic temperature sensor using evanescent fields in D fibers," IEEE Photonics Technol. Lett. 17(12), 2706-2708 (2005)

23. S. M. Chandani, A. Kulpa, and N. A. F. Jaeger, "Non-destructive determination of cladding thickness in D fibers," IEEE Photonics Technol. Lett. 18(9), 1082-1084 (2006).

24. C. Yeh, "Elliptical dielectric waveguides," J. Appl. Phys. 33(11), 3235-3243 (1962)

25. R. B. Dyott, Elliptical Fiber Waveguides, p. 44, Artech House, Boston (1995).

26. http://www.soes.soton.ac.uk/research/groups/oil_monitor/oiltype.htm

27. A. C. García and J. E. Cuena, "Transduction techniques based on intensity modulation of light," Chap. 11 in Handbook of Optical Fiber Sensing Technology, J. M. López-Higuera, Ed., pp. 221-223, Wiley, West Sussex, United Kingdom (2002).

28. B. E. Jones, R. S. Medlock, and R. C. Spooncer, "Intensity and wavelength-based sensors and optical actuators," Chap. 12 in Optical Fiber Sensors: Systems and Applications Volume 2, B. Culshaw and J. Dakin, Eds., pp. 433-434, Artech House, Norwood (1989).

29. A. T. Andreev and K. P. Panajotov, "Distributed single-mode fiber to single-mode planar waveguide coupler," J. Lightwave Technol. 11(12), 1985-1989 (1993).

30. W. Johnstone, S. Murray, G. Thursby, M. Gill, A. McDonach, D. Moodie, and B. Culshaw, "Fiber optic modulators using active multimode waveguide overlays," Electron. Lett. 27(11), 894-896 (1991).

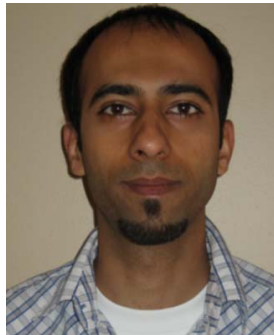

Sameer M. Chandani received the BScE degree in engineering physics from Queen's University, Kingston, Ontario, in 1998, the MASc and $\mathrm{PhD}$ in electrical engineering from the University of British Columbia (UBC), Vancouver, BC, in 2002 and 2007, respectively. From 1998 to 1999 he worked at Gennum Corporation, Burlington, Ontario, as a product development engineer where he was involved in the design of multi-chip electronic modules for hearing aids. His research interests include fiber-optic sensors and electrooptic devices.

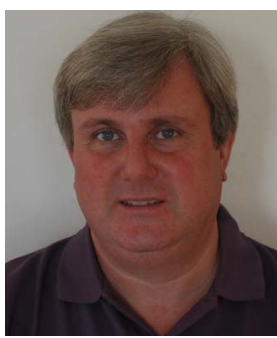

Nicolas A. F. Jaeger received the BSc degree in electrical engineering from the University of the Pacific, Stockton, CA, in 1981 and the MASc and PhD degrees in electrical engineering from the University of British Columbia (UBC), Vancouver, BC, in 1986 and 1989 , respectively. $\mathrm{He}$ is currently a Professor with the Department of Electrical and Computer Engineering, UBC. His research interests include integrated optics, fiber optics, optical sensors and ultrahighspeed modulators and measurement techniques. Professor Jaeger was a recipient of the Canadian Institute of Energy's Research and Development Award, the BC Advanced Systems Institute's Technology Partnership Award, the Natural Sciences and Engineering Research Council of Canada and the Conference Board of Canada's Synergy Award, and the Canadian Association of Physicists and the National Optics Institute's Medal for Outstanding Achievement in Applied Photonics. Professor Jaeger is a member of the SPIE and IEEE. 